evaluations employing a ?hard? outcome such as mortality can help overcome these problems.

Objectives To understand the correlates of depression in RA and to determine whether depression itself is predictive of mortality in RA.

Methods We studied 1500 consecutive RA outpatients during a 20 year period beginning in 1981. 1483 patients completed 27,773 depression questionnaires from the Arthritis Measurement Impact Scales (AIMS). The relation of depression to other variables was studied with generalised estimating equations (GEE). For ease of interpretation, depression was considered present when the AIMS score was 4 or greater in the GEE analyses. The predictability for mortality of depression, and other variables obtained 1) during the first 2 years of follow-up and 2) over the entire course of RA was examined using Cox regression (Cox) models.

Results Depression was related to age $<65$ years (odds ratio 1.3), non-white ethnic origin (OR 1.9), and decreased family income, but was unrelated to sex or marital status. Compared to university graduates, secondary school graduates and non-graduates had increased risk for depression, OR 1.4 and 2.1 respectively. In multivariate models, education, income and ethnic origin were independently related to depression. In clinical models, ESR, white cell count, joint count, grip strength, patient global severity, pain and HAQ (HAQ OR 2.33 (95\% CI 2.11, $2.59, \mathrm{p}<0.001)$ were also strongly related to depression. In univariate Cox models, 2-year depression was predictive of mortality $(\mathrm{z}=3.55 \mathrm{p}<0.001)$, and remained predictive even when ESR, joint count, WBC and grip strength were added as 2-year covariates $(\mathrm{z}=2.00 \mathrm{p}=0.046)$. However, when HAQ disability, patient global, or pain was added to the model, depression was no longer significant $(p=0.388)$. The same results were noted when the 2-year depression scores were modelled with full covariates (from first through last observation). Finally the hazard ratio (HR) for the effect of a depression score of 4 or greater over the full course of the disease was 1.33 (95\% CI $1.09,1.63)$ after adjusting for all covariates. This result was maintained after also controlling for blood pressure, BMI, prednisone use and comorbid illnesses.

Conclusion Depression during the first 2 years of clinical care is associated with increased RA mortality, and is also associated when laboratory or physician associated covariates are added. The effect of depression on mortality is strengthened by followup, and this association (HR 1.33) is maintained even after the inclusion of all covariates. These data suggest that depression has a small but definite association with mortality in RA. Even so, it is possible that additional medical factors that were not recognised by our models might be causally related to depression and therefore to mortality.

\section{THU0125 IS ANXIETY A MORE COMMON DISORDER THAN DEPRESSION IN RHEUMATOID ARTHRITIS?}

${ }^{1}$ YM El Miedany, ${ }^{2}$ AM Haroun. ${ }^{1}$ Rheumatology Department; ${ }^{2}$ Neuropsychiatry Department, Ain Shams University, Faculty of Medicine, Cairo, Egypt

10.1136/annrheumdis-2001.1027

Background Most of the previous clinical researches published payed more attention to study depressive disorders and/or symptoms in patients with rheumatoid arthritis (RA). Fewer research studies were done to investigate the prevalence of anxiety in patients with RA patients.
Objectives This study was performed to investigate whether anxiety disorder is as common as depressive disorder in RA patients and to look for the sociodemographic as well as the clinical characteristics of the patients developing these disorders. Methods A detailed physical assessment of 80 patients with RA was performed. Also psychiatric assessment was done using Research Diagnostic Criteria for the International Classification of Disease (ICD-10). In addition, patients answered a Health Assessment Questionaire (HAQ) to assess their functional capacity.

Results Depression was diagnosed in $66.25 \%$ of the total sample while anxiety was diagnosed in $70 \%$. Functional disability, social stress and morning stiffness were the factors highly associated with depression. Using multiple regression analysis, anxiety was also highly associated with depression. On the other hand, Ritchie articular index as well as depression were positively associated with anxiety disorder.

Conclusion Psychiatric illness is a relatively common disorder in patients with RA, with frequency higher than that of other general medical conditions. Anxiety is a more common disorder than depression. The first step in correct management is recognition of anxiety and depression so that appropriate treatment can be tried. Particular attention has to be paid to social stress and lack of social support.

\section{THU0126 DEXTERITY AND ANXIETY IN LARGE COHORT OF RHEUMATOID ARTHRITIS PATIENTS}

SJ Stropuviene, J Jankauskiene, J Dadoniene, A Venalis. Department of Rheumatology, Institute of Experimental and Clinical Medicine, Vilnius, Lithuania

\subsection{6/annrheumdis-2001.1028}

Background Psychological distress is often a leading condition in rheumatoid arthritis clinical picture in addition to disability and disease activity measures. Being long lasting process it makes an impact on disease coping process and management itself. ${ }^{1}$

Objectives The aim of the study was to determine which demographic and clinical variables relate to anxiety and depression most of all in 225 rheumatoid arthritis (RA) patients cohort.

Methods The study was performed as satellite project to Vilnius rheumatoid arthritis register. The patients were invited to participate in the study and to answer the questions concerning education and employment, physical phunctioning: ACR functional classes, dependence on relatives and HAQ and patients global assessment of health status. The disease activity was measured by physicians global assessment, pain VAS, morning stiffness and modified DAS. Anxiety and depression were measured by AIMS2 questioner particularly asking about mood alterations with range of possible answers: 1-never and 6-almost ever. The level 6,0 for anxiety and 4,0 for dexterity were used as cut of levels. ${ }^{2}$ The chi-square method was used for evaluation of associations and was qualified as significant if $\mathrm{p}<0,05$.

Results In this particular cohort we found that $32 \%$ of patients were experiencing the anxiety and even 42,2\% - depressive moods. As the result, we found strong associations between anxiety levels with disease outcome measures: physical functioning classes (chi2 $=12,72 ; \mathrm{p}=0,00)$, disability indices (chi2 = $21,09 ; \mathrm{p}=0,00)$ and employment holding (chi2 $=5,62 ; \mathrm{p}=$ $0,01)$. Global evaluation of disease activity by physician was the only one significantly correlated disease activity measure (chi2 = $12,51 ; \mathrm{p}=0,00)$. Dexterity levels showed the same significant associations with the same measures. In addition to it dexterity 
had one more additional significant association: the dependence on relatives made those patients evidently depressed (chi2 $=$ $12,96 ; \mathrm{p}=0,00)$ but not anxious.

Conclusion The anxiety and depression status of $225 \mathrm{Ra}$ patients turned out to be multidependent variables in strong association with social status, disability indices and ACR classes but physicians global assessment as the only one disease activity measure.

\section{REFERENCES}

1 Meenan RF, Gertman PM, Mason JH, Dunaif R. The arthritis impact measures scales. Arthritis Rheum. 1982;25:1048-53

2 Hawley DJ, Wolfe F. Anxiety and depression in patients with rheumatoid arthritis: a prospective study of 400 patients. J Rheumatol. 1988;15:932-41

\section{THU0127 THE EVALUATION OF DISEASE OUTCOME MEASURES IN 225 RHEUMATOID ARTHRITIS LITHUANIAN PATIENTS}

JJ Jankauskiene, S Stropuviene, J Dadoniene, A Luksiene, A Jurgelenas. Rheumatology Department, The Institute of Experimental and Clinical Medicine, Vilnius, Lithuania

\subsection{6/annrheumdis-2001.1029}

Background It is commonly recognised that outcome measures in rheumatoid arthritis depend on the duration of disease and especially disability index reveals that link while the others demonstrate minor changes in the course of disease.

Objectives To test the latter hypotheses we performed the study which aimed to measure long-term outcomes of rheumatoid arthritis in 225 patients - Vilnius city inhabitants.

Methods The patients were asked to fill in the self-administered questioners as follows: Stanford HAQ (0-3,3 worth health), AIMS(1-6, 6 worth), SF-36 (0-100, 0 worth health). The entire cohort was subdivided into three groups depending on the disease duration. The first cohort with the short disease less than 5 years encountered 70 patients, second cohort between 5 to 10 years of disease duration encountered 56 patients and 99 patients with long standing disease more than 10 years duration consisted the third cohort. The differences between means (SD) of the variables were measured by F-ratio and qualified as significant if $\mathrm{p}<0.05$.

Results The significant differences between the cohorts were found in disability indices measured by health assessment questioner: $1,21(0,75), 1,17(0,69)$ and $1,45(0,83)$ respectively with F-ratio higher than critical value $(\mathrm{p}=0,048)$. Aims depression and anxiety measures were not significantly different between the cohorts, the same as quality of life indices measured by SF36. The study was found to be powerful enough $-0,7$ - to reveal the possible differences.

Conclusion The only differences between disease duration cohorts were revealed in measuring disability indices which tended to grow with increasing disease duration while the other measures were not sensitive to change. This large enough study showed that psychological distress and quality of life measures do not depend on disease duration and the disease impact is particularly visible on disease indices.

\section{THU0128 SYSTEMIC AA AMYLOIDOSIS IN RHEUMATOID ARTHRITIS - THE INFLUENCE OF AGE, SEX, ONSET AND DURATION OF DISEASE. A RETROSPECTIVE STUDY}

MI Bely, A Apathy. Pathology, Policlinic of the Hospitaller Brothers of St. John of God in Budapest, Budapest, Hungary
Background Rheumatoid arthritis (RA) is a chronic, recurrent autoimmune inflammatory disease, which may be complicated, like other chronic inflammatory diseases, by systemic secondary (reactive) AA amyloidosis (AAa). The precursors of AA amyloid protein fibrils are produced by the liver. During the progressive, cumulative process of systemic AAa the serum amyloid A proteins spread via the blood-stream and deposit throughout the body. AAa in its early stage involves only a few tissue structures (blood vessels, collagen, and reticulin fibres, basal membranes, etc.) in some organs, and increasingly more in its later stages. In advanced stages of AAa massive amyloid deposits in the kidney lead to renal insufficiency and the patients die of uremia. The extension and quantity of amyloid deposits depend on the production, and/or elimination (resolution, excretion) of amyloid proteins, or from the pathologist's point of view on the stage of this progressive disease at death.

Objectives The aim of this study was to determine the influence of age, sex, onset of RA, and disease duration on amyloidosis.

Methods A randomised autopsy population of 161 in-patients (female 116, average age of 64.9 years; male 45, average age of 66.2 years at death) with rheumatoid arthritis (RA) was studied.

The existence of amyloidosis and extent of amyloid deposits at death was determined histologically.

The average amount of amyloid deposition in various structures was visually evaluated on a 0 to 4 plus scale.

The correlations were determined by Mann-Whitney (Wilcoxon), critical random check, Student (Welch) t-probe, and by $c^{2}$-tests, comparing the age, sex, onset of RA, and duration of disease at the time of death: with or without amyloidosis, mild (? <2? per patient) and severe amyloidosis (?2

Results Systemic secondary (AA) amyloidosis was observed in 34 (21.1\%) of 161 cases: in 21 cases amyloidosis was, and 13 it was severe. There was no significant difference of survival time (age at death), onset of RA, and disease duration between males and females, or between patients with and without amyloidosis. RA started earlier, the disease duration was longer, and the age of the patients at death was significantly lower $(\mathrm{p}<0.005)$ in cases with severe amyloidosis, in comparison to those with mild amyloidosis.

Conclusion Amyloidosis may develop in both sexes, and may start at any time during RA. AAa existed in different stages (mild: characterised by sporadic and minimal amyloid deposits, or severe: characterised by diffuse and massive amyloid deposits) at the time of death. There were no significant differences in disease duration and survival time between males and females, or between patients with and without amyloidosis, suggesting that amyloid deposition may occur at different rates. AAa may develop as a rapidly progressive, or as a chronic, slowly progressive disease. Possibly, the process may become inactive (quiescent), or even regress at some point in time.

The risk of severe amyloidosis $(2<)$ is higher in patients with early onset and with long duration of RA, and in these patients the age at death is significantly lower $(\mathrm{p}<0.005)$.

\section{THU0129 UNAVOIDABLE AND MODIFIABLE RISK FACTORS FOR ISCHAEMIC HEART DISEASE (IHD) IN RHEUMATOID ARTHRITIS (RA)}

${ }^{1} \mathrm{NX}$ Erb, ${ }^{1} \mathrm{MJ}$ Banks, ${ }^{2} \mathrm{FF}$ Rowe, ${ }^{1} \mathrm{GD}$ Kitas. ${ }^{1}$ Department of Rheumatology, Dudley Group of Hospitals NHS Trust, Dudley; ${ }^{2}$ The High Field Rheumatology Unit, Worcestershire Acute Hospitals NHS Trust, Worcester, UK 